\title{
Article \\ A FAST DATA PROCESSING TECHNIQUE FOR CONTINUOUS GRAVITATIONAL WAVE SEARCHES
}

\author{
Massimo Tinto ${ }^{1,2}$ (D0000-0002-8107-5148
}

1 University of California San Diego, Center for Astrophysics and Space Sciences, 9500 Gilman Dr, La Jolla, CA 92093, U.S.A.

2 Divisão de Astrofísica, Instituto Nacional de Pesquisas Espaciais, S. J. Campos, SP 12227-010, Brazil; mtinto@ucsd.edu

\begin{abstract}
This article discusses the potential advantages of a data processing technique for continuous gravitational wave signals searches in the data measured by ground-based gravitational wave interferometers. Its main advantage over other techniques is that it does not need to search over the signal's direction of propagation. Although it is a " coherent method" (i.e. it coherently processes year-long data), it is applied to a data set obtained by multiplying the original timeseries with a (half-year) time-shifted copy of it. As a result, the phase modulation due to the interferometer motion around the Sun is automatically canceled in the signal of the synthesized time-series. Although the resulting signal-to-noise ratio is not as high as that of a coherent search, it equals that of current hierarchical methods. In addition, since the signal search is performed over a parameters space of smaller dimensionality, the associated false-alarm probability should be smaller than those characterizing hierarchical methods and result in an improved likelihood of detection.
\end{abstract}

Keywords: Gravitational waves; Interferometers; Data Analysis; Continuous gravitational wave signals;

\section{Introduction}

The first direct observation of a gravitational wave (GW) signal announced by the LIGO project [1] on February 11, 2016 [2], represents one of the most important achievements in experimental physics today, and marks the beginning of GW astronomy [3]. By simultaneously measuring and recording strain data with two interferometers at Hanford (Washington) and Livingston (Louisiana), LIGO scientists were able to reach an extremely high level of detection confidence and infer unequivocally the GW source of the observed signal to be a coalescing binary system containing two black-holes of masses $M_{1}=36_{-4}^{+5} M_{\odot}$ and $M_{2}=29_{-4}^{+4} M_{\odot}$ at a luminosity distance of $410_{-180}^{+160} \mathrm{Mpc}$ corresponding to a red-shift $z=0.09_{-0.04}^{+0.03}$ (the above uncertainties being at the 90 percent confidence level).

The network of the two United States LIGO interferometers could constrain the direction to this binary system only to a broad region of the sky because the Italian-French VIRGO detector [4] was not operational at the time of the detection and no electromagnetic counterparts could be uniquely associated with the observed signal. Ground-based observations inherently require use of multiple detectors widely separated on Earth and operating in coincidence. This is because a network of GW interferometers operating at the same time can (i) very effectively discriminate a GW signal from random noise and (ii) provide enough information for reconstructing the parameters characterizing the wave's astrophysical source (such as its sky-location, luminosity distance, mass(es), dynamic time scale, etc.) $[3,5,6,7,8]$.

Since February 2016 several other detections have been announced by LIGO and VIRGO $[9,10,11,12]$, including observations of coalescing binary systems containing two 
neutron stars [13]. The first of such mergers was observed by the LIGO-VIRGO network and corroborated by gamma-ray observations [14]. The LIGO-VIRGO network can uniquely reconstruct the location of the source in the sky [6] because it can rely on two independent time-delays and take advantage of the asymmetry of the detector's antenna patterns w.r.t. the plane containing the three detectors locations. The two points in the sky determined by the two independent time-delays are the mirror-image of each other w.r.t. this plane. Since the detectors antenna patterns are not symmetric w.r.t. this plane, the double-degeneracy from the time-delays can be broken and the correct location of the GW source can be reconstructed.

Searches for continuous GW signals conducted so far by both projects could only identify upper-limits for the amplitudes characterizing these signals $[12,15,16]$. Continuous GW signals in the band accessible by ground interferometers are expected to be emitted by rapidly spinning neutron stars (such as millisecond pulsars) characterized by non-axisymmetric rotations. Although the physical mechanisms supporting large degrees of non-axisymmetry have been assumed to be well understood and the resulting estimated gravitational wave amplitudes had given confidence on their detections $([17,18,19,20])$, a combination of instrument sensitivity improvements together with additional data processing techniques might be required to achieve a successful observation.

This article discusses the potential advantages of a data processing techniques that does not require searching over the signal's direction of propagation. Although this technique is coherent, it is applied to a data set obtained by multiplying the original data with a time-shifted (about half-year) copy of it. As a result its associated signal-to-noise ratio is not as high as that of coherent searches. However, it equals that characterizing existing hierarchical methods and its associated false-alarm probability is further reduced as the signal search is performed over a parameter space of reduced dimensionality over those used by current searches. An outline of this article is given below. In Sec.( 2) we present a general description of the problems associated with the searches of continuous GW signals in the data recorded by ground-based interferometers. After showing that the phase modulation of a sinusoidal gravitational wave signal measured by an interferometer is equal in magnitude and opposite in sign for samples that are six-months apart, we demonstrate that the product of a year-long data set with a copy of itself that is time-shifted by six-months results in a new data set in which the phase of the resulting GW signal no longer depends on the signal's source sky location. In Sec.( 3) we then discuss the statistical quantities characterizing this technique. We note that its associated signal-to-noise ratio is equal in magnitude to that characterizing a typical hierarchical method, while its false-alarm probability should actually be smaller. In Sec.( 4) we then summarize our results and present our conclusions.

\section{Continuous GW Signals Searches}

The GW signals emitted by millisecond pulsars are continuous, narrow band, and quasi-sinusoidal, and can be modeled by a family of parametrized template waveforms. The long observation times, needed to guarantee their detection by optimal filtering, also require to account for temporal changes of the wave's frequency due to the pulsar's spin-down effect and the motion of the detector relative to the Solar System Barycenter (SSB) (which can be regarded as an inertial reference frame). To effectively account for these frequency modulations in the analysis of the data and maximize the likelihood of detection, an accurate model of the GW signal measured by a detector is required. This model is characterized by a set of parameters defining the instantaneous phase of the 
signal measured at the detector, $\Phi(t)$, and the corresponding instantaneous frequency $f(t) \equiv \frac{1}{2 \pi} \frac{d \Phi}{d t}$. Their expressions can be written in the following forms [21]

$$
\begin{aligned}
& \Phi(t)=2 \pi f_{0}\left[t+\frac{\vec{r}(t) \cdot \hat{n}}{c}+\frac{f_{1}}{2}\left(t+\frac{\vec{r}(t) \cdot \hat{n}}{c}\right)^{2}\right], \\
& f(t)=f_{0}\left(1+\frac{\vec{v}(t) \cdot \hat{n}}{c}\right)\left[1+f_{1}\left(t+\frac{\vec{r}(t) \cdot \hat{n}}{c}\right)\right],
\end{aligned}
$$

where $f_{0}$ is the signal frequency at the pulsar's rest frame, $f_{1}$ is the first pulsar's frequency spin-down term, ${ }^{1} \hat{n}$ is the unit vector to the pulsar's sky position relative to the SSB, $(\vec{r}(t)$, $\vec{v}(t))$ are the position and velocity at time $t$ of the detector relative to the SSB respectively, and $c$ is the vacuum speed of light. The spin-down parameter $f_{1}$ can take values in the interval $\left|f_{1}\right|<1 / \delta$, where $\delta$ is the characteristic time scale over which the fractional relative change of the pulsar spin is of the order of unity. If we now denote with $(\theta, \phi)$ the two polar angles associated with the pulsar's sky location, the set of parameters (in principle unknown) characterizing the above two functions and over which a search is performed can be represented by a vector $\vec{\lambda} \equiv\left(f_{0}, f_{1}, \theta, \phi\right)$.

Although the GW strain measured at the interferometer, $s(t)$, is a linear combination of the wave's two polarization components of the form

$$
s(t, \vec{\lambda})=A(t) \cos \left[\Phi(t, \vec{\lambda})+\Phi_{0}\right],
$$

it can been shown that the time-dependence of the amplitude $A$ and phase $\Phi_{0}$ is due to the detector's beam-pattern functions through its orientation to the source. As these two functions change gradually over the course of a day, their frequency components are outside the band of interest and can therefore be treated as constants. This fact has been known for some time in the literature $([21,22,23])$, where it was also estimated that by applying matched-filtering under this assumption would result in a few percents loss in SNR over the case accounting for their time dependence.

The most sensitive search method relies on coherent matched filtering the data against a bank of templates, whose parameters are chosen from the physical space of interest; this yields the detection statistics commonly known in the field as the F-statistics $[22,24]$. This method is, however, computationally intensive when processing year-long data sets over a large parameter space. Since any signal present in the data will possess parameters that are different from any one we might include in the template search bank, it becomes essential to identify a criterion by which the bank is selected so that the maximum degradation in SNR would still result in a detection. The current adopted approach to select the templates, needed to search for a GW signal characterized by a set of parameters, is to introduce a metric in the parameter space to quantify how closely the templates must be spaced [23]. This approach, when applied to searches for continuous signals, results in a number of templates that rapidly increases with the length of the data being analyzed. If we denote with $N$ the total number of samples of the data analyzed, it has been shown [22] that the number of templates placed on the $(\theta, \phi)$ surface scales roughly as $N^{2}$, while that needed for detecting the signal frequency $f_{0}$ and the spin-down parameter $f_{1}$ grow as $N$ and roughly as $N^{2}$ respectively. In other words, the number of templates for an all-sky search scales approximately as $N^{5}$. Since the matched filtering SNR only grows as $N^{1 / 2}$, it is clear that matched filtering quickly becomes computationally impossible with year-long data sets [25].

A solution to this problem, suggested in the literature and implemented in the related LIGO/VIRGO pipelines $[15,16,21,26,27]$, is to use hierarchical algorithms. In this approach the data is decomposed in $n$ segments, each typically containing $m$ data

1 For sake of simplicity, but without loss of generality, our analysis will include only the first derivative of the variation of the pulsar's angular velocity. 
samples so that $N=n \times m$. Each segment is processed by matched filtering separately and then they are all combined using a semi-coherent technique [21,26]. Semi-coherent methods are less sensitive than coherent matched filtering as their resulting SNR scales roughly as $n^{1 / 4} m^{1 / 2}=(m N)^{1 / 4}$. On the other hand, since it would be computationally impossible to coherently search a year-long data set, by breaking it down in "chunks" with a number of samples $m \ll N$ the resulting number of templates needed is reduced significantly and the processing of the data becomes computationally feasible. A number of different semi-coherent algorithms have been developed, and their descriptions can be found in the latest LIGO/VIRGO publications on the subject $[15,16,27]$ and references therein.

\section{The Proposed Technique}

In what follows we highlight a new data processing technique to search for continuous signals in the data generated by ground interferometric detectors such as LIGO and VIRGO. This technique takes advantage of the symmetry of the motion of the interferometer as it rotates around the Sun and simultaneously around the Earth's axis. For sake of illustration of the idea we will first present our data processing technique by disregarding the eccentricity, $e$, of the Earth's trajectory around the Sun and the pulsar's spin-down, $f_{1}$. Later on we will revise this analysis and include both $e$ and $f_{1}$.

Since the velocity of the interferometer w.r.t. the SSB at time $t$ is equal in magnitude and opposite in sign to the value it acquires at a later time $t+T$ (with $T \sim 6$ months), and because the position of the interferometer at time $t+T, \vec{r}(t+T)$, is equal to $-\vec{r}(t)+\vec{\xi}$ (where $\vec{\xi}$ is a constant vector of integration), the expression of the detector's GW response at these two times can be written as

$$
\begin{aligned}
s(t) & =A \cos \left[2 \pi f_{0}\left[t+\frac{\vec{r}(t) \cdot \hat{n}}{c}\right]+\Phi_{0}\right] \\
s(t+T) & =A \cos \left[2 \pi f_{0}\left[t+T-\frac{\vec{r}(t) \cdot \hat{n}}{c}\right]+\Phi_{0}+\Psi_{0}\right],
\end{aligned}
$$

where we have denoted with $\Psi_{0}$ the constant associated with the integrations of the anti-symmetric condition fulfilled by the velocity vector at times $t$ and $t+T$. If we now multiply the two data streams, after some simple algebra we find the following expression for the GW signal in the resulting new time-series

$$
\begin{aligned}
Q^{g w}(t) & \equiv s(t) s(t+T) \\
& =\frac{A^{2}}{2}\left[\cos \left(2 \pi f_{0}[2 t+T]+2 \Phi_{0}+\Psi_{0}\right)+\cos \left(4 \pi f_{0} \frac{\vec{r}(t) \cdot \hat{n}}{c}-T-\Psi_{0}\right)\right]
\end{aligned}
$$

The GW signal in the new time-series is equal to the sum of two terms, one of which no longer depends on the parameters $(\theta, \phi)$ associated with the pulsar's sky location and is purely monochromatic with frequency $2 f_{0}$. In other words, if there were no eccentricity and spin-down effects, such a signal component could be detected in the new time-series by performing a single Fourier transform!

A non-zero eccentricity $e$, a non-negligible pulsar's spin-down $f_{1}$, together with the presence of quadratic noise terms in the newly formed time-series, temper the above analysis and require additional considerations. Let's first focus on the noise terms. Let $R_{k} \equiv R\left(t_{k}\right)=s_{k}+\eta_{k}$ be the detector measurement at time $t_{k}$, with $\eta_{k}$ representing the random process associated with the detector's noise and $k=1, \ldots N$. By multiplying the 
detector's data at time $t_{k}$ with itself at time $t_{k}+N / 2 \Delta t$ (with $\Delta t$ being the sampling time) we now find ${ }^{2}$

$$
Q_{k} \equiv R_{k} R_{k+N / 2}=s_{k} s_{k+N / 2}+s_{k} \eta_{k+N / 2}+s_{k+N / 2} \eta_{k}+\eta_{k} \eta_{k+N / 2} .
$$

In what follows we will assume the noise auto-correlation time to be significantly smaller than $T$ and $\eta$ to be a stationary, Gaussian distributed random process of zero-mean and variance $\sigma_{\eta}^{2}$. Note that the expression for $Q_{k}$ in Eq. (7) tells us that the noise affecting this newly synthesized data set is no longer Gaussian because of the presence of quadratic noise terms.

We now define our detection statistics to be the Fourier transform of $Q_{k}$

$$
S_{k} \equiv \sum_{j=1}^{N / 2} Q_{j} e^{\frac{4 \pi i k j}{N}}
$$

where $i$ is the imaginary unit. Since the Fourier transform is typically taken over a relatively large number of points, the probability distribution of the noise entering the statistics defined by Eq. (8) becomes Gaussian as a result of the central limit theorem. This means that it is entirely characterized by its mean and variance, which may be obtained by estimating the expectation value of $S_{k}, \mu_{S}$, and its quadratic deviation from its mean, $\sigma_{S}^{2}$. By denoting with $l$ the integer corresponding to the frequency $2 f_{0}$, it is easy to find the following expressions for the mean and variance associated with the above statistics

$$
\begin{aligned}
\mu_{S} & \equiv\left\langle S_{l}\right\rangle=\sum_{j=1}^{N / 2} s_{j} s_{j+N / 2} e^{\frac{4 \pi l j}{N}} \simeq \frac{A^{2}}{4}\left(\frac{N}{2}\right) \\
\sigma_{S}^{2} & \left.\left.\equiv\langle| S_{l}-\mu_{S}\right)\left.\right|^{2}\right\rangle \simeq \sigma_{\eta}^{4} \frac{N}{2}
\end{aligned}
$$

where we have denoted with $\langle\ldots\rangle$ the operation of expectation value. The expression above for $\sigma_{S}^{2}$ reflects the observation that GW amplitudes emitted by pulsars are expected to be much smaller than the noise level; also we assumed an integration time equal to the data off-set time $T \simeq 6$ months $=N / 2 \times \Delta t$. From Eqs. $(9,10)$ we can then derive the expression of the amplitude SNR achievable by our data processing technique

$$
S N R \equiv \frac{A}{2 \sigma_{\eta}}\left(\frac{N}{2}\right)^{1 / 4} .
$$

Note the SNR dependence on the fourth-root power of the number of data samples. Although such dependence is worse than that achievable by a fully-coherent search (for which the SNR grows as the square-root of the number of samples), it is well known that a fully-coherent search of GW signals from an isolated pulsar whose parameters are unknown is computationally impossible [22]. Although the dependence of the SNR on the fourth-root power of the integration time is also characteristic of hierarchical, semi-coherent methods implemented by ground interferometers, our approach should offer the advantage of achieving detection without searching over the pulsar's position on the celestial sphere. This should result in a reduced false-alarm probability and therefore an improved sensitivity.

2 Although the samples required to construct the non-linear combination given in Eq. (7) might not exists, it is possible to construct them with exquisite accuracy by applying Fractional-Delay Filtering (FDF) to the data points from the surrounding samples [28,29]. 
To complete our analysis, in what follows we first include the pulsar's spin-down parameter $f_{1}$. The expression of $Q^{g w}(t)$ with $f_{1} \neq 0$ is now equal to

$$
\begin{aligned}
Q^{g w}(t) & =\frac{A^{2}}{2}\left\{\operatorname { c o s } \left(2 \pi f _ { 0 } \left[2 t+T+\frac{f_{1}}{2}\left(2 t^{2}+2 t T+T^{2}-2 T \frac{\vec{r}(t) \cdot \hat{n}}{c}\right.\right.\right.\right. \\
& \left.\left.\left.+2\left(\frac{\vec{r}(t) \cdot \hat{n}}{c}\right)^{2}\right)\right]+2 \Phi_{0}+\Psi_{0}\right)+\cos \left(2 \pi f _ { 0 } \left[2 \frac{\vec{r}(t) \cdot \hat{n}}{c}-T\right.\right. \\
& \left.\left.\left.+\frac{f_{1}}{2}(2 t+T)\left(2 \frac{\vec{r}(t) \cdot \hat{n}}{c}-T\right)\right]-\Psi_{0}\right)\right\} .
\end{aligned}
$$

The GW signal in the new time-series is again equal to the sum of two terms. Although now the first term shows a dependence on the parameters $(\theta, \phi)$, such a dependence is nevertheless suppressed by the spin-down parameter $f_{1}$. This suggests that, if we would break the new time-series in a number $n$ of contiguous data chunks of duration $\tau$ properly selected, we could neglect the signal's dependence on the sky position. The time duration, $\tau$, during which we can neglect the dependence over the sky-position parameters, is given by the following inequality relating the frequency shift experienced by the gravitational wave signal in $Q^{g w}(t)$ to the frequency resolution $\tau^{-1}$

$$
f_{0} f_{1} T \frac{\vec{v}(t) \cdot \hat{n}}{c} \leq \tau^{-1} .
$$

The above expression includes only the larger of the two quantities depending on $(\theta, \phi)$ entering the first term of the signal expression given in Eq.(12).

To quantify the possible values of $\tau$ compatible with typical source parameters, we will first consider two classes of pulsars introduced in [23]: (i) young, fast pulsars, with $f_{0}<1000 \mathrm{~Hz}$ and $f_{1}<1 / \delta=1 / 40 \mathrm{yr}^{-1}$, and (ii) old, slow pulsars, with $f_{0}<200 \mathrm{~Hz}$ and $f_{1}<1 / \delta=1 / 1000 \mathrm{yr}^{-1}$. In the case of "young \& fast" pulsars, we find $\tau \leq 800 \mathrm{sec}$ after taking a maximum Doppler shift of $10^{-4}$; "slow \& old" pulsars instead would require a $\tau \leq 10^{5} \mathrm{sec}$. On the other end, if we would limit the intervals of definition for $f_{0}$ and $f_{1}$ to those adopted by the latest low-frequency searches for continuous signals in the LIGO and VIRGO data [27] $\left(f_{0} \epsilon(20,128) \mathrm{Hz}\right.$ and $\left.f_{1} \epsilon\left(-1.0 \times 10^{-10} / f_{0}, 1.5 \times 10^{-11} / f_{0}\right)\right) \mathrm{Hz}$, we would conclude that there would not be any need to break up the data since $\tau>T$.

We will now quantify the effect of the Earth's eccentricity by focusing on the velocities at the apogee and perigee (exactly six months apart). Although at these two points the signs of the velocities are exactly opposite, their magnitudes are not and they differ the most at these points. Their percent difference is in fact equal to $2 e \simeq 3.4 \times 10^{-2}$. The corresponding frequency shift, which now depends on the source location parameters and is proportional to $f_{0}$, could be as large as $3.2 \times 10^{-3} \mathrm{~Hz}$ for a signal with frequency $f_{0}=1 \mathrm{kHz}$. This in turn implies that if we would take chunks of data shorter than about $300 \mathrm{sec}$. we could still disregard searching for the location of a source emitting a continuous signal of frequency $f_{0}=1 \mathrm{kHz}$ in the $n$ chunks of the constructed time-series. This frequency value of course reflects the worst possible interferometer's geometrical configuration associated with the apogee/perigee points. To avoid or further minimize the effects associated with the eccentricity, the reference frame of our coordinate system could be moved away from the SSB to the center of the elliptical trajectory of the Earth around the Sun. Also, the time shift $T$ could be treated as a slowly varying function of time so as to make the velocities at times $t$ and $t+T$ (away from the apogee/perigee) as "close" as possible.

Finally, if the break-up of the data in $n$ chunks results necessary, one can regain SNR by "stacking-up" the $n$ spectra. Since the noise in each set is uncorrelated with all the others, it is easy to show that the resulting amplitude SNR is equal to

$$
S N R \equiv \frac{A}{2 \sigma_{\eta}}\left(\frac{N}{2}\right)^{1 / 4} \text {. }
$$


after using the identity $n \times m=N$.

\section{Discussion}

The proposed data processing technique has the appealing feature of not requiring a search over the parameters $(\theta, \phi)$ associated with the source location. This gain, however, results in a SNR that grows as $N^{1 / 4}$ rather than $N^{1 / 2}$ characteristic of coherent searches. Nevertheless, the SNR dependence of our technique on the data length is equal to that shown by semi-coherent searches. Since this is achieved by requiring a significantly smaller number of templates due to a smaller-size parameter space, it offers some interesting advantages worth further investigation. At the exploratory level we have conducted so far, such a technique should result not only in faster searches, but also in a reduced false-alarm probability due to the smaller number of parameters searched for. This should translate in an improved sensitivity over other non-coherent methods.

Acknowledgments: It is a great pleasure to thank the Center for Astrophysics and Space Sciences (CASS) at the University of California San Diego (UCSD, U.S.A.) and the National Institute for Space Research (INPE, Brazil) for their kind hospitality while this work was done.

[1] Aasi, J.; et. al.. Advanced LIGO. Class. Quantum Grav. 2015, 32, 074001. doi:10.1088/0264-9381/32/7/074001.

[2] Abbott, B.P.; et. al.. Observation of Gravitational Waves from a Binary Black Hole Merger. Phys. Rev. Lett. 2016, 116, 061102. doi:10.1103/PhysRevLett.116.061102.

[3] Thorne, K.S. Gravitational Radiation. In 300 Years of Gravitation; Hawking, S.; Israel, W., Eds.; Cambridge University Press: Cambridge, 1987.

[4] Accadia, T.; et. al.. Virgo: a laser interferometer to detect gravitational waves. J. Instrum. 2012, 7, P03012. doi:10.1088/17480221/7/03/P03012.

[5] Schutz, B.F.; Tinto, M. Antenna patterns of interferometric detectors of gravitational waves - I. Linearly polarized waves. Monthly Notices of the Royal Astronomical Society 1987, 224, 131-154. doi:10.1093/mnras/224.1.131.

[6] Gürsel, Y.; Tinto, M. Near optimal solution to the inverse problem for gravitational-wave bursts. Phys. Rev. D 1989, 40, 3884-3938. doi:10.1103/PhysRevD.40.3884.

[7] Sathyaprakash, B.S.; Dhurandhar, S.V. Choice of filters for the detection of gravitational waves from coalescing binaries. Phys. Rev. D 1991, 44, 3819-3834. doi:10.1103/PhysRevD.44.3819.

[8] Jaranowski, P.; Krolak, A. Optimal solution to the inverse problem for the gravitational wave signal of a coalescing compact binary. Phys. Rev. D 1994, 49, 1723-1739. doi:10.1103/PhysRevD.49.1723.

[9] Abbott, B.P.; et al.. GW151226: Observation of Gravitational Waves from a 22-Solar-Mass Binary Black Hole Coalescence. Phys. Rev. Lett. 2016, 116, 241103. doi:10.1103/PhysRevLett.116.241103.

[10] Abbott, B.P.; et al.. GW170104: Observation of a 50-Solar-Mass Binary Black Hole Coalescence at Redshift 0.2. Phys. Rev. Lett. 2017, 118, 221101. doi:10.1103/PhysRevLett.118.221101.

[11] Abbott, B.P.; et al.. GW170814: A Three-Detector Observation of Gravitational Waves from a Binary Black Hole Coalescence. Phys. Rev. Lett. 2017, 119, 141101. doi:10.1103/PhysRevLett.119.141101.

[12] Abbott, B.P.; et al.. GWTC-1: A Gravitational-Wave Transient Catalog of Compact Binary Mergers Observed by LIGO and Virgo during the First and Second Observing Runs. Phys. Rev. X 2019, 9, 031040. doi:10.1103/PhysRevX.9.031040.

[13] Abbott, B.P.; et al.. GW170817: Observation of Gravitational Waves from a Binary Neutron Star Inspiral. Phys. Rev. Lett. 2017, 119, 161101. doi:10.1103/PhysRevLett.119.161101.

[14] Abbott, B.P.; et al.. Gravitational Waves and Gamma-Rays from a Binary Neutron Star Merger: GW170817 and GRB 170817A. The Astrophysical Journal Letters 2017, 848, L13.

[15] Abbott, B.P.; et al.. All-sky search for periodic gravitational waves in the O1 LIGO data. Phys. Rev. D 2017, 96, 062002. doi:10.1103/PhysRevD.96.062002.

[16] Abbott, B.P.; et al. First low-frequency Einstein@Home all-sky search for continuous gravitational waves in Advanced LIGO data. ArXiv e-prints: https://arxiv.org/abs/1707.02669 2017, [arXiv:gr-qc/1707.02669].

[17] Prix, R. Search for continuous gravitational waves: Metric of the multidetector $\mathcal{F}$-statistic. Phys. Rev. D 2007, 75, 023004. doi:10.1103/PhysRevD.75.023004.

[18] Sathyaprakash, B.S.; Schutz, B.F. Physics, Astrophysics and Cosmology with Gravitational Waves. Living Reviews in Relativity 2009, 12, 2. doi:10.12942/lrr-2009-2.

[19] Owen, B.J. Maximum Elastic Deformations of Compact Stars with Exotic Equations of State. Phys. Rev. Lett. 2005, 95, 211101. doi:10.1103/PhysRevLett.95.211101.

[20] Johnson-McDaniel, N.K.; Owen, B.J. Maximum elastic deformations of relativistic stars. Phys. Rev. D 2013, 88, 044004. doi:10.1103/PhysRevD.88.044004. 
[21] Brady, P.R.; Creighton, T. Searching for periodic sources with LIGO. II. Hierarchical searches. Phys. Rev. D 2000, 61, 082001. doi:10.1103/PhysRevD.61.082001.

[22] Jaranowski, P.; Królak, A.; Schutz, B.F. Data analysis of gravitational-wave signals from spinning neutron stars: The signal and its detection. Phys. Rev. D 1998, 58, 063001. doi:10.1103/PhysRevD.58.063001.

[23] Brady, P.R.; Creighton, T.; Cutler, C.; Schutz, B.F. Searching for periodic sources with LIGO. Phys. Rev. D 1998, 57, $2101-2116$. doi:10.1103/PhysRevD.57.2101.

[24] Cutler, C.; Schutz, B.F. Generalized $\mathcal{F}$-statistic: Multiple detectors and multiple gravitational wave pulsars. Phys. Rev. D 2005, 72, 063006. doi:10.1103/PhysRevD.72.063006.

[25] Jaranowski, P.; Królak, A. Gravitational-Wave Data Analysis. Formalism and Sample Applications: The Gaussian Case. Living Reviews in Relativity 2012, 15, 4. doi:10.12942/lrr-2012-4.

[26] Astone, P.; Colla, A.; D'Antonio, S.; Frasca, S.; Palomba, C. Method for all-sky searches of continuous gravitational wave signals using the frequency-Hough transform. Phys. Rev. D 2014, 90, 042002. doi:10.1103/PhysRevD.90.042002.

[27] Aasi, J.; et al. First low frequency all-sky search for continuous gravitational wave signals. Phys. Rev. D 2016, $93,042007$. doi:10.1103/PhysRevD.93.042007.

[28] Laakso, T.I.; Valimaki, V.; Karjalainen, M.; Laine, U.K. Splitting the unit delay [FIR/all pass filters design]. IEEE Signal Processing Magazine 1996, 13, 30-60. doi:10.1109/79.482137.

[29] Shaddock, D.A.; Ware, B.; Spero, R.E.; Vallisneri, M. Postprocessed time-delay interferometry for LISA. Phys. Rev. D 2004, 70, 081101. doi:10.1103/PhysRevD.70.081101. 